\title{
FORMATION OF A POLAR STRUCTURE IN THE METALLIC NIOBIUM SULFIDE NB $\mathrm{S}_{3}$
}

Fabian Grahlow, a Eric Dorsch, ${ }^{\text {b }}$ Markus Ströbele, ${ }^{\text {a }}$ Carl P. Romao*a and Hans-Jürgen Meyer*a

a Section for Solid State and Theoretical Inorganic Chemistry, Institute of Inorganic Chemistry, Auf der Morgenstelle 18, Eberhard Karls Universität Tübingen, D-72076 Tübingen, Germany

b Physikalisches Institut, Auf der Morgenstelle 14, Eberhard Karls Universität Tübingen, D-72076 Tübingen, Germany

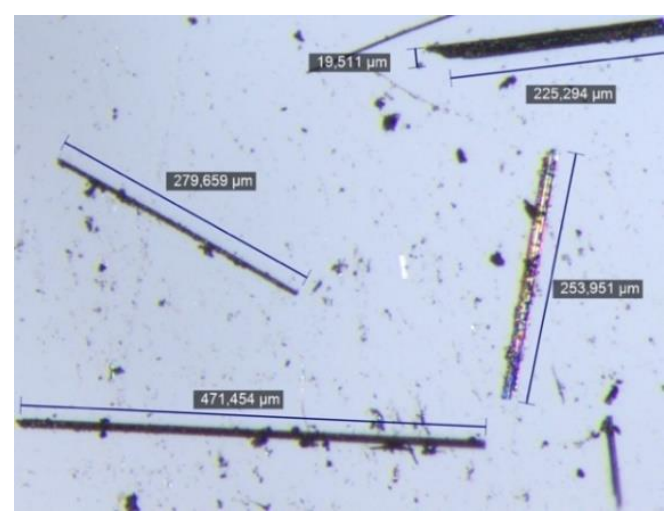

Figure S1. Optical micrograph of $\mathrm{Nb}_{4} \mathrm{~S}_{3}$ crystals.

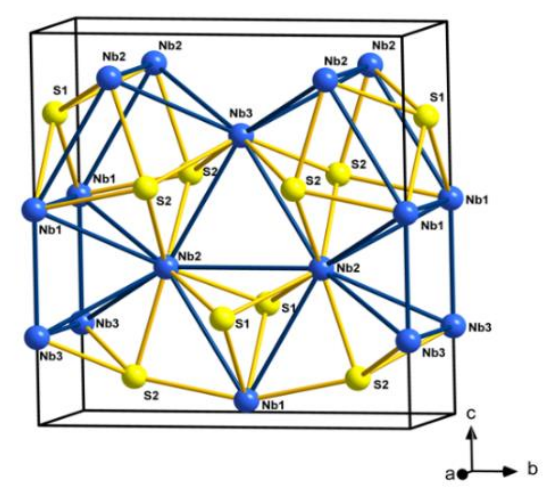

Figure S2. Unit cell of the crystal structure of $\mathrm{Nb}_{4} \mathrm{~S}_{3}$.

Table S1. Mass of Educts used for the synthesis of $\mathrm{Nb}_{4} \mathrm{~S}_{3}$.

\begin{tabular}{lllll}
\hline Reactants & $\mathrm{NbBr}_{5}$ & $\mathrm{Nb}$ & $\mathrm{LiBr}$ & $\mathrm{S}$ \\
Mass [mg] & 67.7 & 19.1 & 9.9 & 3.6 \\
\hline
\end{tabular}

Table S2. Interatomic distances in different niobium sulfides in $\AA$.

\begin{tabular}{llll}
\hline Niobium sulfides & $\mathbf{N b}-\mathbf{S}[\AA \AA]$ & $\mathbf{N b}-\mathbf{N b}[\AA]$ & $\mathbf{S} \sim \mathbf{S}[\mathbf{A}]$ \\
\hline $\mathrm{Nb}_{21} \mathrm{~S}_{8}$ & $2.44-2.55$ & $2.88-2.95$ & $3.26-3.36$ \\
$\mathrm{Nb}_{14} \mathrm{~S}_{5}$ & $2.45-2.71$ & $2.76-3.37$ & $3.55-3.70$ \\
$\mathbf{N b}_{4} \mathbf{S}_{3}$ & $\mathbf{2 . 4 8 - 2 . 5 3}$ & $\mathbf{2 . 7 7 - 3 . 3 4}$ & $\mathbf{3 . 2 1 - 3 . 5 5}$ \\
$\mathrm{NbS}$ & 2.51 & $3.23-3.32$ & $3.23-3.76$ \\
$\mathrm{Nb}_{3} S_{4}$ & $2.46-2.61$ & 2.88 & $2.88-3.37$ \\
$\mathrm{NbS}_{2}$ & 2.47 & 3.33 & $3.11-3.45$ \\
$\mathrm{NbS}_{3}$ & $2.43-2.67$ & 3.04 & 2.05 \\
\hline
\end{tabular}

Table S3. Anisotropic displacement parameters $\left(\AA^{2} \times 1^{3}\right)$ for $\mathrm{Nb}_{4} S_{3}$. The anisotropic displacement factor exponent takes the form: $2 \pi^{2}\left(h^{2} a^{* 2} U^{11}+\ldots+2 \pi a^{*} b^{*} U^{12}\right)$

\begin{tabular}{lllllll}
\hline & $\mathrm{U}^{11}$ & $\mathrm{U}^{22}$ & $\mathrm{U}^{33}$ & $\mathrm{U}^{23}$ & $\boldsymbol{U}^{13}$ & 0 \\
$\mathrm{Nb}(1)$ & $2(1)$ & $2(1)$ & $2(1)$ & 0 & $\boldsymbol{U}^{12}$ & 0 \\
$\mathrm{Nb}(2)$ & $2(1)$ & $3(1)$ & $2(1)$ & $0(1)$ & 0 & 0 \\
$\mathrm{Nb}(3)$ & $2(1)$ & $2(1)$ & $2(1)$ & 0 & 0 & 0 \\
$\mathrm{~S}(1)$ & $2(1)$ & $3(1)$ & $3(1)$ & 0 & 0 & 0 \\
$\mathrm{~S}(2)$ & $2(1)$ & $3(1)$ & $2(1)$ & $0(1)$ & 0 & 0 \\
\hline
\end{tabular}




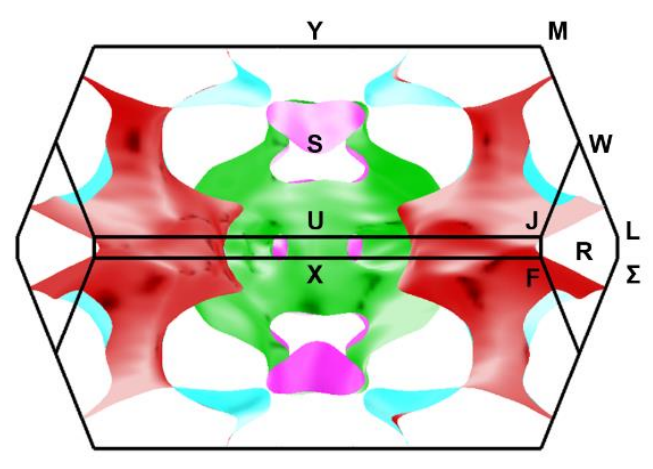

Figure S4. The calculated Fermi surface of $\mathrm{Nb}_{4} \mathrm{~S}_{3}$.

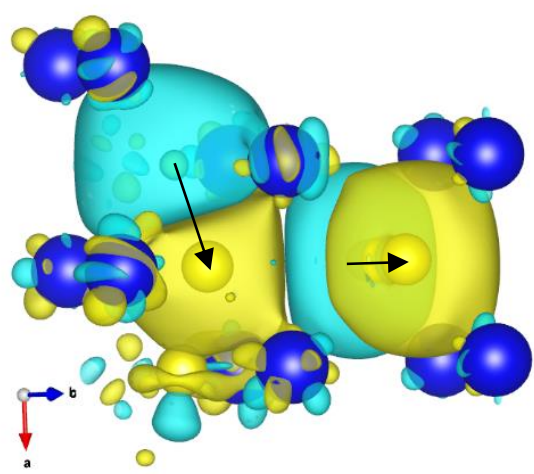

Figure S5. Differences in electron density (shown as isosurfaces at the $0.005 \mathrm{e} /$ bohr $^{3}$ (yellow) and $-0.005 \mathrm{e} / \mathrm{bohr}^{3}$ (cyan) levels) following the displacement (black arrows) of an S(1) and an S(2) atom from pseudo-nonpolar to polar positions.

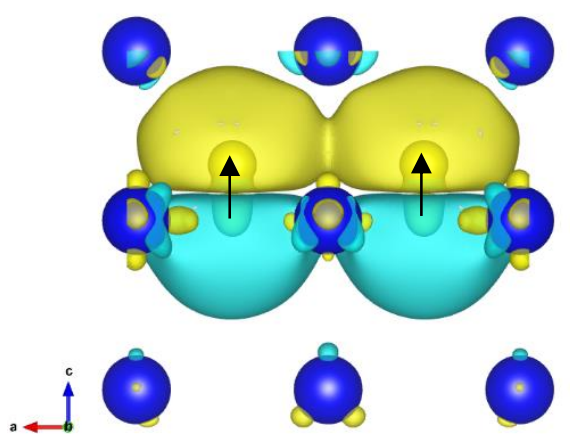

Figure S6. Differences in electron density (shown as isosurfaces at the $0.005 \mathrm{e} / \mathrm{bohr}^{3}$ (yellow) and $-0.005 \mathrm{e} / \mathrm{bohr}^{3}$ (cyan) levels) following the displacement (black arrows) of two S(1) atoms from pseudo-nonpolar to polar positions.

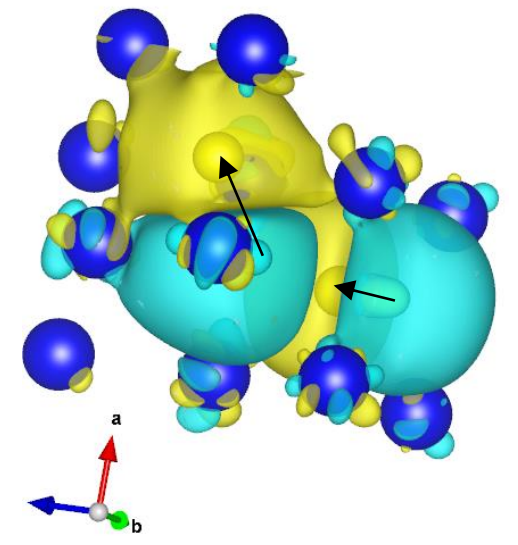

Figure S7. Differences in electron density (shown as isosurfaces at the $0.005 \mathrm{e} / \mathrm{bohr}^{3}$ (yellow) and $-0.005 \mathrm{e} / \mathrm{bohr}^{3}$ (cyan) levels) following the displacement (black arrows) of an $S(1)$ and an $S(2)$ atom from pseudo-nonpolar to polar positions. 